\title{
TEXTUAL DEVICES
}

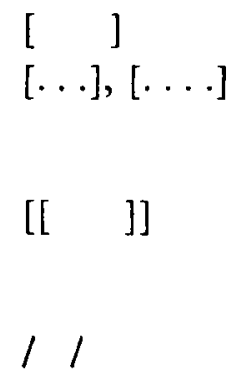

[roman]

[italic]

eanceled.
Blank spaces in the text.

Suspension points indicate approximate number of letters or digits missing in words or numerals (not to exceed four) and not conjecturable.

Double square brackets are used to give the composition date of a published letter or news report if the publication date differs.

Incorporation into the text of addition or correction made above or below the line by author.

Conjectural reading for missing, mutilated, or illegible matter, with a question mark inside the square bracket when the conjectural reading is doubtful. Also used in editorial correction of typographical errors in original manuscript or printed document. Also used to indicate the publication date of a news report or periodical article.

Assigned date of any undated document; editorial comment inserted in the text, such as [endorsement], [illegible], [remainder missing], [sentence unfinished], [torn], [enclosure], [attachment]; [in the margin].

Textual matter deleted in the original but restored in the text. 
